\title{
Metagenomic analysis of intestinal microbiota in geese from different farming systems in Gunungpati, Semarang
}

\author{
R Susanti ${ }^{1 *}$, Ari Yuniastuti ${ }^{1}$, Fitri Arum Sasi ${ }^{1}$, Muchamad Dafip ${ }^{2}$ \\ ${ }^{1}$ Department of Biology, Faculty of Mathematics and Natural Sciences, Universitas Negeri Semarang, Sekaran Gunungpati, Semarang, Jawa \\ Tengah 50229, Indonesia \\ ${ }^{2}$ Master Degree Program in Biomedical Sciences, Faculty of Medicine, Universitas Diponegoro, Jl. Prof. Sudarto SH, Tembalang, Semarang, \\ Jawa Tengah 50275, Indonesia \\ *Corresponding author: r.susanti@mail.unnes.ac.id
}

SUBMITTED 31 January 2020 REVISED 4 May 2020 ACCEPTED 7 July 2020

\begin{abstract}
The diversity of intestinal bacteria in geese correlates with environmental conditions, rearing methods, and consumed feeds. The intestinal bacteria composition is useful for the absorption of nutrition, improving the metabolism, and may be related to the immune system. This study was conducted to examine the intestinal bacteria composition and the diversity of maintained goose in aviaries and barns. This research was an observational exploratory. Five geese were taken purposively from local breeders in Gunungpati District, Semarang City. A total of $5 \mathrm{~g}$ of intestinal contents from each sample was used for microbial genome isolation. Then, the genome was amplified to collect $16 \mathrm{~S}$ rRNA gene region V3-V4. The amplicons were then sequenced using the next generation sequencing (NGS) method (Illumina high-throughput sequencing; paired-end reads) and analyzed using QIIME2 to identify bacterial species. In addition, GC-MS was performed to identify and measure fatty acid contents in the intestinal. The results showed that both rearing and caged goose contained nine phyla of intestinal bacteria. The number of intestinal bacteria of barn geese (SU) reached 32,748 Operational Taxonomy Units (OTU); higher than aviary geese (SK), which was 11,646 OTU. The intestinal bacteria community in barn geese was approved by Phylum TM7 (Saccharibacteria candidate) (53.18\%), followed by Firmicutes (32.51\%) and Bacteriodetes (5.42\%). Whereas on SK Firmicutes was compiled $49.34 \%$ of total OTU, TM7 (S. candidate) up to $21.17 \%$, and Actinobacteria up to $15.99 \%$. The abundance of TM7 may contribute to high 9,12-octadecadienoic acid production, while Firmicutes was related to the high production of oleic acid. Based on these data, the reared geese had a more abundant diversity of bacteria than the caged one.
\end{abstract}

KEYWORDS Aviary; barn; goose; intestinal bacteria; metagenomics; rearing management

\section{Introduction}

Intestinal microorganisms are a bacterial community that settles inside the intestinal tract of living things, including geese. The intestinal bacteria composition is influenced by environmental conditions, feed and the host's internal body condition. It directly affects metabolism, absorption of nutrients, physiology, and immune regulation (Altizer et al. 2011). Goose is a waterfowl that can be an influenza virus reservoir in their digestive tract (Zhou et al. 2006; Harris et al. 2010; Kim 2018). However, it does not trigger to a severe condition of viral-infection-diseases (Susanti et al. 2018). The ability to be a virus reservoir may be related to intestinal bacteria (Phuong et al. 2011; Mandl et al. 2015). In other case, an imbalance of microhabitat conditions of intestinal bacterial or dysbiosis has been proven to increase the risk of diseases in the digestive system. It may actively contribute to food vulnerability in the future (Altizer et al. 2011). It makes the geese play an essential role in preventing outbreaks of infectious disease pandemics. At present, the studies related to this case have not been focused on many researchers. Therefore, efforts to examine the role of the intestinal bacteria in geese as a reservoir of disease agents must be continuously investigated.

The rearing pattern is well known as one of the mechanisms for controlling intestinal microbiome conditions. Both method, aviary (caged geese) and barn (free-living) cultivations, may contribute directly to the commensal and pathogenic bacteria in the intestinal (Leung and Koprivnikar 2016). Previous research from (DominguezBello et al. 2010) showed that the barn goose has more diverse and abundant intestinal bacteria comparing to the aviary. Free-range or barn geese also produced a high quality of meat and healthier (Yamak et al. 2016).

The metagenomic approach is the most appropriate technique for determining the composition of bacteria, both in the environment and the digestive tracts of geese. This technique is the basis for understanding the intesti- 
nal bacterial taxonomy, which helps determine which one is the best cultivation method based on the condition of intestinal bacteria. By analyzing the metagenome condition, it will be easier to identify various bacteria species. Some uncultured bacteria in the intestinal tract are impossible to identification using the conventional polymerase chain reaction (PCR) technique. Based on the previous research, it is necessary to study the microbiota of goose intestines to analyze the composition of intestinal bacteria and the metabolism quality of aviary and barn farming systems related to host immunity.

\section{Materials and Methods}

This research was an exploratory observational study to analyze the composition, abundance of intestinal bacteria, and its correlations with feed and environmental conditions. Aviary geese (SK) and barn geese (SU) samples were collected from the local community's goose husbandry in Gunungpati Subdistrict, Semarang. Samples were obtained purposively with sampling criteria (inclusions), i.e. aviary and barn geese, males or females aged at least three months, did not receive feed or drugs containing antibiotics within two weeks. Samples obtained were excluded from the study sample if known to be laying eggs.

In this study, the aviary geese (the caged geese) were fed only concentrates, grains, and leftovers from household food waste. Then, barn geese farming was in the broader foraging area, and it may increase the intake of various types of food and nutrients from the environment, especially root and leave as plant fiber sources. However, despite having a different maintenance pattern, both the aviary and barn geese were always getting additional pellets fed in, including mixed with rice bran, rice, and corn.

\subsection{Sample preparation}

Five female-geese, which consisted of three geese from the aviary and two geese from the barn farming system, were collected. The geese were sacrificed by slaughtering according to the farmer's standard procedure, then as much as $5 \mathrm{~g}$ intestinal contents were taken aseptically from each goose for two groups. The intestinal content of the waterfowl sample was mixed per each group and homogenized using a vortex. After that, samples were collected in 3 $\mathrm{mL}$ microtubes and frozen at $-20^{\circ} \mathrm{C}$ until further NGS and GC-MS analysis.

\subsection{DNA isolation and next generation sequencing (NGS) analysis}

The microbial genome was extracted from intestinal contents samples using the QIAamp DNA Stool Mini Kit (Qiagen, San Diego, California, US) according to the manufacturer's protocol. The extracted DNA was stored in the $-20{ }^{\circ} \mathrm{C}$ freezer. Species identification was performed by amplifying $16 \mathrm{~S}$ rRNA genome in the V3-V4 region for accurate and precise results (Dennis et al. 2013; Yarza et al. 2014). The amplification process was run using Illumina HiSeq 2500 platform for 20 cycles according to a procedure by Holm et al. (2019). The primers used were forward-primer (5'-ACTCCTRCGGGAGGCAGCAG-3') and reverse-primer (5'-GGACTACHVGGGTWTCTAAT3') (Holm et al. 2019).

\subsection{Bioinformatic analysis}

The 16S sequence metagenomic analysis was performed using QIIME2 (Ver. 2019.4) (Caporaso et al. 2010). Paired-end files were demultiplexed using the demux plugin. Then, quality control was performed on each sample using the Dada2 plugin (Callahan et al. 2016). Furthermore, the diversity index value was generated using six diversity indexes: Shannon (Shannon and Weaver 1949), Simpson (Simpson 1949), Pielou evenness (Pielou 1966), Margalef (Magurran 2004), Chao1 (Chao 1984), and Observed OTU's (DeSantis et al. 2006). A taxonomic arrangement was made based on the Greengenes-13_8 99\% OTU database (McDonald et al. 2012), heatmap compilation (Hunter 2007) using the heatmap plugin, and taxa barplot preparation using Microsoft Excel 2010.

\subsection{GC-MS analysis}

Predicted bacteria-side-product short-chain fatty acids (SCFAs) were analyzed using gas chromatography-mass spectrometry (GC-MS). A total of $20 \mathrm{~g}$ of intestinal content was dried at room temperature for three days to reduce water content, then $250 \mathrm{mg}$ of sample was taken, and mashed using a mortar-pestle and wrapped in filter paper and placed in the Soxhlet apparatus extraction tool. The sample was dissolved in $250 \mathrm{~mL}$ of $\mathrm{n}$-hexane to extract the fatty acid group components. The Soxhlet extraction procedure was conducted by modification steps from Eneroth et al. (1968) and Batta et al. (2002), and repeated for 12 cycles for each sample.

The extracted intestinal content samples that had been obtained were evaporated at $68{ }^{\circ} \mathrm{C}$ for $6 \mathrm{~h}$ to get their constant weight before being injected into the GC-MS tool. The GC-MS process was performed using Shimadzu QP2010S with AGILENTTJ\% W HP-5 column (30 m long, ID $0.25 \mathrm{~mm}$ ) and helium as the carrier gas. MS operating conditions were as follows: ionization induced by electrons (EI) at $70 \mathrm{eV}$ and ion source temperature up to $250{ }^{\circ} \mathrm{C}$. This compound was identified by conducting a library search using Shimadzu NIST / WILEY + mass spectral database.

\section{Results and Discussion}

\subsection{Result}

Based on the analysis, both aviary (SK) and barn (SU) geese were maintained in a closed-area or did not mixed with other waterfowl or poultry species. However, the foraging area of barn goose was more extensive than the aviary. Therefore, it can be seen that aviary geese consume more starch than plant fibers. 


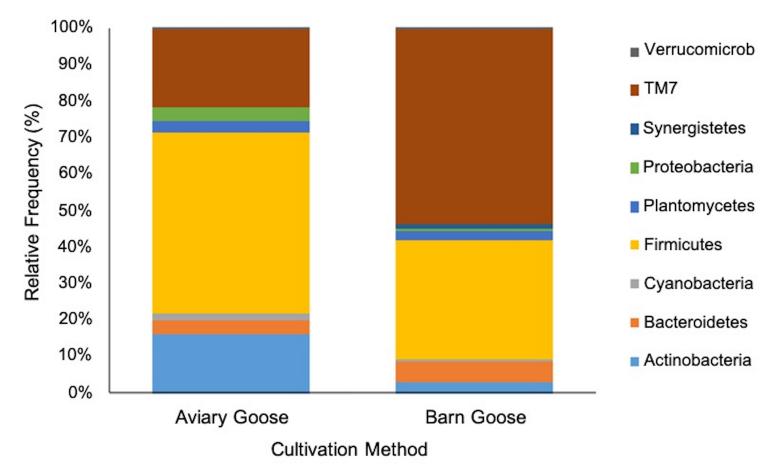

FIGURE 1 Composition of goose intestinal microbiome (Phylum taxa).

Intestinal bacteria can utilize the food substrate to modulate the goose's digestive and immune systems' development and function. Instead, bacteria get habitat and nutrients for growth. The composition of intestinal bacteria is influenced by feed intake. Interaction between various bacteria in the intestinal can also increase growth and reduce the risk of enteric infection by pathogens.

The metagenomic analysis results showed differences in the diversity and abundance of intestinal bacteria between aviary and barn geese. There were at least nine phyla that have been identified from intestinal content. The abundance of reared goose intestinal microbiome reached 32,748 Operational Taxonomy Unite (OTU), more than the goose caged (11,646 OTU). The relative composition of each phylum is shown in Figure 1. In SU geese, intestinal bacteria were dominated by Phylum TM7 (53.18\%), followed by Firmicutes (32.51\%) and Bacteriodetes (5.42\%). Six other phyla (Actinobacteria, Cyanobacteria, Planctomycetes, Proteobacteria, Synergistetes, and Verrucomicrobia) were in the range of 0.69$3.11 \%$. Whereas in SK, intestine colonies dominated by Phylum Firmicutes (49.34\%), followed by TM7 of 21.17\%

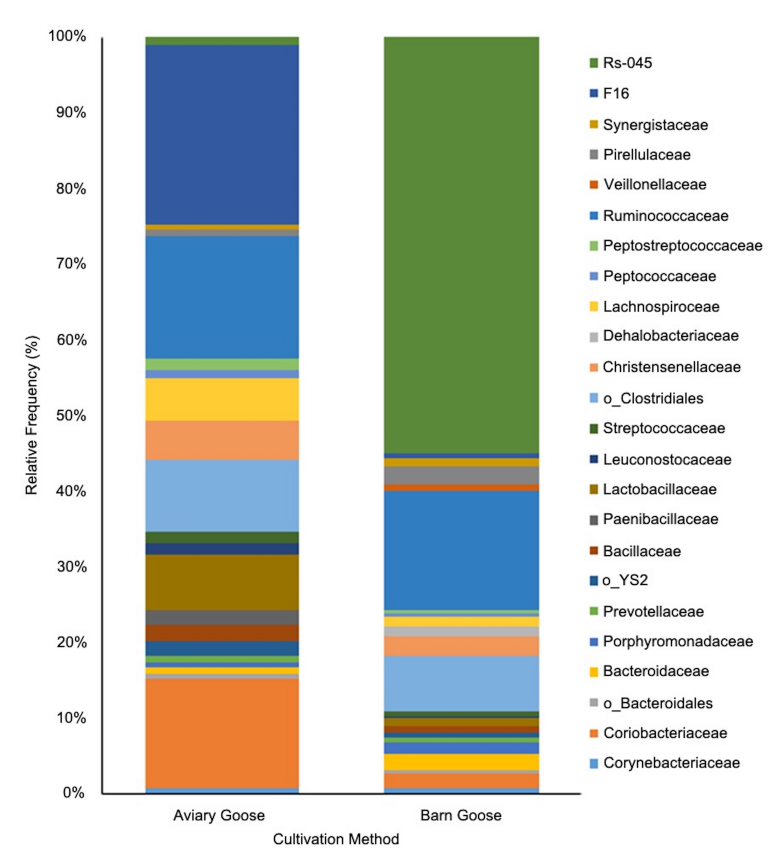

FIGURE 2 Composition of goose intestinal microbiome (Family taxa).

and Actinobacteria 15.99\%. Six other phylum (Bacteriodetes, Cyanobacteria, Planctomycetes, Proteobacteria, Synergistetes, and Verrucomicrobia) were in the range of $0.43-3.94 \%$. Although the diversity of intestinal bacteria in SU individuals was abundant, most phylum (Cyanobacteria, Planctomycetes, Proteobacteria, and Verrucomicrobia) only have a composition $<1.00 \%$ of total intestinal bacteria. While in SK, the composition of almost all bacterial phylum, except Synergistetes and Verrucomicrobia, was more than 1.00

At the lower taxonomic levels, aviary goose's bacteria were dominated by the F16 family (23.09\%), followed by Ruminococcaceae (16.16\%), Coriobacteriaceae (14.42\%), Clostridiales (9.55\%), Lactobacillaceae (7.25\%), Lach-

TABLE 1 Index of diversity of goose intestinal bacteria.

\begin{tabular}{llllll}
\hline \multirow{2}{*}{ Sample } & \multicolumn{5}{c}{ Diversity index } \\
\cline { 2 - 6 } & Shannon & Simpson & Margalef & Chao1 & Pielou evenness \\
\hline Barn geese (SU) & 5.67 & 0.94 & 18.42 & 174 & 0.76 \\
Aviary gees (SK) & 3.72 & 0.72 & 14.39 & 151 & 0.51 \\
\hline
\end{tabular}

TABLE 2 Data on the results of GCMS analysis of goose intestine contents.

\begin{tabular}{|c|c|c|c|c|c|}
\hline Sample & RT & High & Area & \%area & Components \\
\hline \multicolumn{6}{|c|}{ Aviary goose (SK) } \\
\hline SK1 & 8,296 & $2,83 E+08$ & 19121908 & $25.41 \%$ & Hexadecanoic acid \\
\hline SK2 & 8,826 & 67691040 & 2710253,8 & $3.6 \%$ & 9-octadecenoic acid (Z)-methyl ester \\
\hline SK3 & 9,111 & $4,58 \mathrm{E}+08$ & 53429756 & $70.99 \%$ & Oleic Acid \\
\hline \multicolumn{6}{|c|}{ Barn goose (SU) } \\
\hline SU1 & 8,296 & $1,96 \mathrm{E}+08$ & 19121908 & $25.41 \%$ & Hexadecanoic acid \\
\hline SU2 & 9,101 & $3,04 \mathrm{E}+08$ & 41681548 & $77.17 \%$ & 9,12-octadecadienoic acid (Z,Z)- \\
\hline
\end{tabular}


K_Bacteria; P_Firmicutes; C_Clostrida; O_Clostridiales; F_Dehalobacteriaceae; G_Dehalobacterium; $S_{-}$

K_Bacteria; P_Firmicutes;C_Clostrida;O_Clostridiales; F_Veillonellaceae; G_Meganomonas; S $_{\text {_ }}$

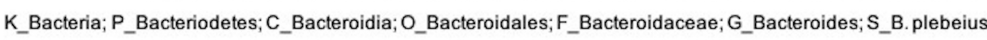
K_Bacteria; P_Actinobacteria; C_Actinobacteria; O_Actinomycetales; F_Corynobacteriaceae; G_Corynebacterium; $S_{-}$

K_Bacteria; P_Firmicutes; C_Clostrida; O_Clostridiales; F_Veillonellaceae; G_Phascolarctobacterium; S $_{-}$

K_Bacteria; $P_{-}$Proteobacteria; $C_{-}$Gammaproteobacteria; $O_{-}$Enterobacteriales; $F_{-}$Enterobacteriaceae; $G_{-} ; S_{-}$ K_Bacteria; P_TM7; CTM7; O_CW040; F_F16; G_; S

K_Bacteria; P_Verrucomicrobia;C_Verrucomicrobiae; O_Verrucomicrobiales; $F_{-}$Verrucomicrobiaceae; G_Akkermansia;

K_Bacteria; P_Bacteriodetes; C_Bacteroidia; O_Bacteroidales; F_Bacteroidaceae; G_Bacteroides; S_B. barnesiae

K_Bacteria; P_Firmicutes;C_Clostrida;O_Clostridiales; F_Lachnospiraceae; G_[Ruminococcus]; S_

K_Bacteria; P_Firmicutes; C_Bacilli; O_Bacillales; F_Bacillaceae;G_Bacillus; $S_{-}$

K_Bacteria; $P_{-}$Bacteriodetes; $C_{-} B a c t e r o i d i a ; O_{-}$Bacteroidales; $F_{-} ; G_{-} ; S_{-}$

K_Bacteria; P_Bacteriodetes; C_Bacteroidia; O_Bacteroidales; F_Prevotellaceae; G_Provotella; $S_{-}$ K_Bacteria; P_Bacteriodetes; C_Bacteroidia; O_Bacteroidales; F_Ruminococcaceae; G_Butyricicoccus; S_B. pullicaecorum

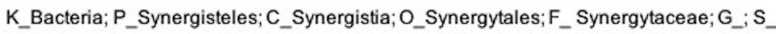

K_Bacteria; $P_{-}$Bacteroidetes; C_Bacteroidia; O_Bacteroidales; F_Bacteroidaceae; G_Bacteroides; $S_{-}$ K_Bacteria; P_Bacteroidetes; C_Bacteroidia; O_Bacteroidales; F_Porphyromonadaceae; G_Parabacteroied; S $_{\text {_ }}$

K_Bacteria; P_Actinobacteria;C_Coriobacteria; O_Coriobacteriales; F_Coriobacteriaceae; G_Slackia; S_ K_Bacteria; P_Actinobacteria;C_Coriobacteria; O_Coriobacteriales; F_Coriobacteriaceae; G_Collinsella; _ $_{\text {_ }}$

K_Bacteria; P_Firmicutes; C_Bacilli; O_Lactobacillales; F_Lactobacillaceae; $G_{-} ; S_{-}$

K_Bacteria; P_Firmicutes; C_Bacilli; O_Lactobacillales; F_Lactobacillaceae; G_Lactobacillus; $S_{\text {_ }}$

K_Bacteria; P_Actinobacteria;C_Coriobacteriia; O_Coriobacteriales; $F_{-}$Coriobacteriaceae; $G_{-} ; S_{-}$ K_Bacteria; P_Firmicutes; C_Clostrida; O_Clostridiales; F_Ruminococcaeae; G_Gemmiger; S_G. formicilis K_Bacteria; $P$ _Firmicutes;C_Clostrida; O_Clostridiales; F_Veillonellaceae; G_Phascolarctobacterium; $S_{-}$

K_Bacteria; P_Firmicutes; C_Clostridia; O_Clostridiales; F_[Mogibacteriaceae]; G_;

K_Bacteria; $P_{-}$Bacteroidetes; C_Bacteroidia; O_Bacteroidales; $F_{-}$Rikenellaceae; G_; K_Bacteria; P_Firmicutes; C_Bacilli; O_Lactobacillales; F_Streptococcaceae; G_Streptococcus; S_S. Iuteciae

K_Bacteria; P_Firmicutes;C_Clostridia; O_Clostridiales; F_Peptococcaceae;G_; K_Bacteria; P_Firmicutes;C_Clostridia;O_Clostridiales; F_Peptostreptococcaceae; G_[Clostridium];

K_Bacteria; P_Proteobacteria; C_Alphaproteobacteria; O_Rickettsiales; $F_{-} ; G_{-} ; S_{-}$ K_Bacteria; P_Firmicutes; C_Clostridia; O_Clostridiales; F_; G_;

K_Bacteria; P_Firmicutes;C_Clostridia;O_Clostridiales; F_Ruminococcaceae;G_;

K_Bacteria; P_Firmicutes; C_Clostridia; O_Clostridiales; F_Christensenellaceae; G_; K_Bacteria; P_Firmicutes; C_Clostridia;O_Clostridiales; F_; G_;

K_Bacteria; P_Firmicutes;C_Clostridia; O_Clostridiales; F_Ruminococcaceae; G_;

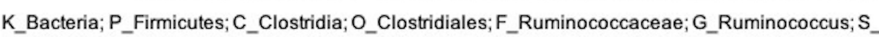
K_Bacteria; P_Firmicutes; C_Clostridia; O_Clostridiales; F_Ruminococcaceae;G_Oscillospira;

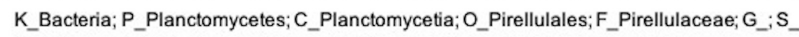
K_Bacteria; P_Actinobacteria;C_Coriobacterila; O_Coriobacteriales; F_Coriobacteriaceae; G_; K_Bacteria; P_Firmicutes; C_Clostridia; O_Clostridiales; F_Lachnospiraceae; $G_{-} ; S_{-}$ K_Bacteria; $P_{-} ; C_{-} ; O_{-} ; F_{-} ; G_{-} ; S_{-}$

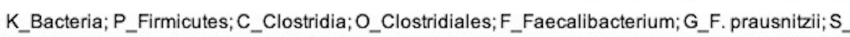
K_Bacteria; P_Actinobacteria; C_Coriobacteriia; O_Coriobacteriales; F_Coriobacteriaceae; G_Adiercreutzia; _ $_{-}$ K_Bacteria; P_Cyanobacteria;C_4COd-2;O_Y52;F_; G_;

FIGURE 3 Heatmap diagram showing the diversity of intestinal bacteria in aviary goose and barn goose. 
nospiraceae (5.52\%), and Christensenellaceae (5.15\%) (Figure 2). Eight families were in the range of 1-3\%, and nine other families were bellowing $1.00 \%$. The intestinal bacteria in barn geese was dominated by Rs-045 family (family groups of Saccharibacteria candidate) (55.04\%), followed by Ruminococcaceae (15.62\%), Clostridiales (7.50\%), and Christensenellaceae (2.47\%). Six families were in the range $1-2.46 \%$, and 12 other families were in the range $<1.00$. The Paenibacillaceae family was only found in the aviary goose $(2.07 \%)$ and was not found in the barn goose. Overall, the barn goose has higher bacteria composition, which was 31,217 OTU; compared to the aviary goose, which was 9,693 OTU.

The composition of intestinal bacteria in barn goose was more diverse than the aviary. It can be seen from the number of species that have not been identified. In aviary goose, approximately $4.78 \%$ of species were not identified, whereas, in barn goose, only around $3.02 \%$ of species were not identified. Of the total bacteria (Figure 1), several OTUs were shared species owned by both aviary and barn goose.

The appearance of intestinal bacterial density shows that some OTUs identified from SK has a darker color than SU (Figure 3). It shows that some bacteria may be abundant in the barn but lower in aviary geese. The diversity shown by the diversity index shows that SU geese have higher variability (Table 1). Overall, there were no anomalies, which are deviations of one or more values to the other values, in the diversity index. It shows that all parameters of the diversity index state that barn goose has higher intestinal bacterial diversity than the aviary.

Intestinal bacteria indirectly play an essential role in regulating host metabolism through the production of vitamin compounds, essential amino acids, and short-chain fatty acids (SCFAs). Therefore, the abundance of intestinal bacteria has an impact on the production of these SCFA. The results of GC-MS analysis showed that the intestinal content was composed of SCFA, hexadecanoic acid, 9-octadecenoic acid (Z)-, methyl ester, and oleic acid. While in barn goose, there were hexadecanoic acid and 9,12-octadecadienoic acid (Z, Z) (Table 2).

\subsection{Discussion}

In general, most people perform geese farming as a source of food and pets. Goose rearing patterns in the communities generally do not develop husbandry on an extensive and intensive (closed) scale, as does the community in $\mathrm{Gu}$ nungpati, Semarang. Goose has a smaller proportion of the digestive tract, so that the transit time for food to be digested is shorter than a mammal. However, based on the analysis of intestinal contents shows that most of the organic fiber has been completely digested (unpublished data). This research focuses on studies related to cultivation techniques, environment-moreover, diversity of goose intestinal bacteria that conducted in Gunungpati, Semarang.

Intestinal bacteria play an essential role in the metabolism of carbohydrates and fibers, proteins, and lipids. Cultivation patterns affecting livestock access in foraging activity. The root content of plants and leaves of grass is the dominant component found in the barn goose's gizzard. It indicates that the grass and the remaining organic material is the leading fiber supply of geese. High fiber is a component of non-starch polysaccharide (NSP) known as the primary material digested or fermented by intestinal bacteria. The fermentation of NSP breakdown products produces short-chain volatile fatty acids (SCFAs) (Jamroz et al. 2002), absorbed by the mucosa and catabolized.

In this study, the most dominant phyla is TM7 in barn goose and Firmicutes in the aviary, followed by Actinobacteria, Bacteroidetes, Planctomycetes, and Proteobacteria, which is in line with a study by Yang et al. (2018). Wang et al. (2016) also showed that Firmicutes, Proteobacteria, Actinobacteria, and Bacteroidetes were the dominant phyla in poultry feces. Furthermore, more microbiota phyla are found in the cecum than in other parts of the intestine.

The domestication process of the goose aims to increases body mass by increasing carbohydrate-based-feed. It increases the Firmicutes colony (Grond 2017). Firmicutes produces SCFA as a by-product of the fermentation process, which can be directly absorbed by the host intestinal cell (den Besten et al. 2013). Several studies have shown a positive relationship between the excess quantity of Firmicutes and metabolic functions. Thus, prebioticcontaining feed, such as Bacillus subtilis and Enterococcus faecium, can increase nutrient uptakes and general metabolic efficiencies (Li et al. 2016; Zheng et al. 2016).

In contrast to the high Firmicutes density in the aviary goose group, barn goose bacteria is dominated by TM7 bacteria. The TM7 is known as a Saccharibacteria candidate that is predicted to be epibiotic parasites growing on the other bacteria surface (He et al. 2015). The increase in the density of Saccharibacteria candidates remains unclear. However, it may cause by maintenance patterns of goose, which the bacteria density increases in the dryland rearing (Zhao et al. 2019) and high cellulose consumption (He et al. 2015). Saccharibacteria candidates are predominantly found in the duodenum (Zhu et al. 2020), where the cellulose remains and not split into smaller components. The polysaccharides, especially the cell wall, mostly degraded by Bacteroidetes (Thomas et al. 2011). However, the abundance Bacteriodetes phyla in waterfowl, especially goose, is still not well understood. It is likely due to differences in diet, and the wide dietary range between waterfowl species (further investigations of this relationship are needed). In this research, the Firmicutes are mostly composed of bacteria from the Clostridia family and may contain pathogenic bacteria Clostridium botulinum, well known as avian botulinum infection agent, but it is needed further analysis to identify the specific pathogenic species.

Bacteroides are an important bacteria related to fibrolysis and or active fermentation of microbial ecosystems in the intestine (den Besten et al. 2013). Bacteroides are thought to be related to this part's specific role in the health 
and performance of poultry through fermentation products, SCFA, with host's genes (Pan and Yu 2014). Also, there are Actinobacteria bacteria that are more dominant in aviary goose than barn goose. The abundance of Actinobacteria is positively correlated with the fiber intake (Dominianni et al. 2015).

The primary function of Proteobacteria in the digestive tract of poultry is not yet certainty known. However, an increase in the quantity and abundance of Proteobacteria groups increased in wild waterfowl species associated with the ocean ecosystem, $57.5 \%$, compared to the terrestrial environment that only 45.5\% (Grond 2017). Proteobacteria are also known to be the most essential intestinal bacteria involved in the degradation of active acid herbicides (Liu et al. 2011), indicating the possible role of detoxification in the digestive tract.

Geese do not have a complete metabolic cycle to metabolize polysaccharides until they are ready to be absorbed. Based on research, as many as $20 \%$ of genes in poultry intestinal bacteria are the genes responsible for polysaccharides metabolism (Thomas et al. 2011; Singh et al. 2014), including major enzymes such as carbohydrate esterase, amylase, and glycoside hydrolase, which less on geese (Beckmann et al. 2006; Yeoman et al. 2012).

Beside, intestinal bacteria provides unique compounds that can improve the quality and viability of duck poultry. During polysaccharide digestion, microbiota produces various kinds of SCFA, mostly acetate, propionate, butylate, valerate, isobutylene, and isovalerate, and vitamin $\mathrm{K}$ (Yeoman et al. 2012). Intestinal bacteria can utilize the food substrate to modulate the development and function of the digestive and immune systems in swan hosts. Instead, the host is a permissive habitat and nutrition for bacterial colonization and growth. Intestinal bacteria can be affected by feed, and usually, different feed interventions are used by feed production to increase goose growth and reduce the risk of enteric infection by pathogens.

\section{Conclusions}

The barn pattern provides more opportunities for the goose to expand the foraging areas, which may increase the intake of various types of organic fibers. Various feed directly contributes to the diversity of intestinal bacteria. It was proved from an abundance of intestinal bacteria in barn goose, which reaches 32,748 Operational Taxonomy Unite (OTU), while aviary goose is 11,646 OTU. It may also correlate with bacteria diversity, which showed that barn geese have a higher diversity score in overall indices. However, the highest bacteria composition in barn geese was Saccharibacteria candidate phylum, followed by Firmicutes, and the opposite in the aviary geese. Meanwhile, the abundance of Saccharibacteria may contribute to high 9,12-octadecadienoic acid production, while Firmicutes is related to the high production of oleic acid. Nevertheless, further study is needed to help in understanding the roles of bacteria in terms of SCFA production.

\section{Acknowledgments}

We thank to Fidia Febriana, M. Sc., for assistance with language proofreading, and comments that greatly improved the manuscript.

\section{Authors' contributions}

This research was constructed by cooperating program includes: Conceptualization, RS and MD; instrument and methodology, RS, MD and FAS.; validation, RS and AY; formal analysis, AY; investigation, RS, FAS amd MD; resources, RS; data curation, AY; writing-original draft preparation, RS and MD; writing-review and editing, $\mathrm{RS}$, and MD; visualization, MD; supervision, RS; project administration, AY; funding acquisition, RS. All authors have read and agreed to the published version of the manuscript.

\section{Competing interests}

There is no conflict of interest with any financial, personal, or other relationships with other people or organization related to the material discussed in the manuscript.

\section{References}

Altizer S, Bartel R, Han BA. 2011. Animal migration and infectious disease risk. Science. 331(6015):296-302. doi:10.1126/science.1194694.

Batta AK, Salen G, Batta P, Tint GS, Alberts DS, Earnest DL. 2002. Simultaneous quantitation of fatty acids, sterols and bile acids in human stool by capillary gas-liquid chromatography. J Chromatogr B Analyt Technol Biomed Life Sci. 775(2):153-161. doi:10.1016/S1570-0232(02)00289-1.

Beckmann L, Simon O, Vahjen W. 2006. Isolation and identification of mixed linked $\beta$-glucan degrading bacteria in the intestine of broiler chickens and partial characterization of respective 1,3-1,4- $\beta$ glucanase activities. J Basic Microbiol. 46(3):175185. doi:10.1002/jobm.200510107.

Callahan BJ, McMurdie PJ, Rosen MJ, Han AW, Johnson AJA, Holman SP. 2016. DADA2: High resolution sample inference from Illumina amplicon data. Nat Methods. 13(7):581-583. doi:10.1038/nmeth.3869.DADA2.

Caporaso JG, Kuczynski J, Stombaugh J, Bittinger $\mathrm{K}$, Bushman FD, Costello EK, Fierer N, Peña AG, Goodrich JK, Gordon JI, et al. 2010. QIIME allows analysis of high-throughput community sequencing data. Nat Methods. 7(5):335-336. doi:10.1038/nmeth.f.303.QIIME.

Chao A. 1984. Nonparametric Estimation of the Number of Classes in a Population. Scand Stat Theory Appl. 11(4):265. 
den Besten G, Van Eunen K, Groen AK, Venema K, Reijngoud DJ, Bakker BM. 2013. The role of shortchain fatty acids in the interplay between diet, gut microbiota, and host energy metabolism. J Lipid Res. 54(9):2325-2340. doi:10.1194/jlr.R036012.

Dennis KL, Wang Y, Blatner NR, Wang S, Saadalla A, Trudeau E, Roers A, Weaver CT, Lee JJ, Gilbert JA, et al. 2013. Adenomatous polyps are drivenbymicrobe-instigated focal inflammation and are controlled by IL-10-producing T cells. Cancer Res. 73(19):5905-5913. doi:10.1158/00085472.CAN-13-1511.

DeSantis TZ, Hugenholtz P, Larsen N, Rojas M, Brodie EL, Keller K, Huber T, Dalevi D, Hu P, Andersen GL. 2006. Greengenes, a chimera-checked 16S rRNA gene database and workbench compatible with ARB. Appl Environ Microbiol. 72(7):5069-5072. doi:10.1128/AEM.03006-05.

Dominguez-Bello MG, Costello EK, Contreras M, Magris M, Hidalgo G, Fierer N, Knight R. 2010. Delivery mode shapes the acquisition and structure of the initial microbiota across multiple body habitats in newborns. Proc Natl Acad Sci USA. 107(26):1197111975. doi:10.1073/pnas.1002601107.

Dominianni C, Sinha R, Goedert JJ, Pei Z, Yang L, Hayes RB, Ahn J. 2015. Sex, body mass index, and dietary fiber intake influence the human gut microbiome. PLoS ONE. 10(4):1-14. doi:10.1371/journal.pone.0124599.

Eneroth P, Hellstrom K, Sjovall J. 1968. A Method for Quantitative Determination of bile acid in human feces. Acta Chem Scand. 22(6):1720-1744.

Grond K. 2017. Development and dynamics of gut microbial communities of migratory shorebirds in the Western Hemisphere. Ph.D. thesis, Kansas State University. doi:10.1017/CBO9781107415324.004.

Harris MT, Brown JD, Goekjian VH, Luttrell MP, Poulson RL, Wilcox BR, Swayne DE, Stallknecht DE. 2010. Canada geese and the epidemiology of avian influenza viruses. J Wildl Dis. 46(3):981-987. doi:10.7589/0090-3558-46.3.981.

He X, McLean JS, Edlund A, Yooseph S, Hall AP, Liu SY, Dorrestein PC, Esquenazi E, Hunter RC, Cheng $\mathrm{G}$, et al. 2015. Cultivation of a human-associated TM7 phylotype reveals a reduced genome and epibiotic parasitic lifestyle. Proc Natl Acad Sci USA. 112(1):244-249. doi:10.1073/pnas.1419038112.

Holm JB, Humphrys MS, Robinson CK, Settles ML, Ott $\mathrm{S}$, Fu L, Yang H, Gajer P, He X, McComb E, et al. 2019. Ultrahigh-Throughput Multiplexing and Sequencing of $>500$-Base-Pair Amplicon Regions on the Illumina HiSeq 2500 Platform. mSystems. 4(1):110. doi:10.1128/msystems.00029-19.

Hunter JD. 2007. Matplotlib: A 2D graphics environment. Comput Sci Eng. 9(3):99-104. doi:10.1109/MCSE.2007.55.

Jamroz D, Jakobsen K, Bach Knudsen KE, Wiliczkiewicz A, Orda J. 2002. Digestibility and energy value of non-starch polysaccharides in young chickens, ducks and geese, fed diets containing high amounts of barley. Comp Biochem Physiol A Mol Integr Physiol. 131(3):657-668. doi:10.1016/S10956433(01)00517-7.

Kim SH. 2018. Challenge for one health: Co-circulation of zoonotic h5n1 and h9n2 avian influenza viruses in egypt. Viruses. 10(3):1-16. doi:10.3390/v10030121.

Leung TL, Koprivnikar J. 2016. Nematode parasite diversity in birds: the role of host ecology, life history and migration. J Anim Ecol. 85(6):1471-1480. doi:10.1111/1365-2656.12581.

Li Y, Xu Q, Huang Z, Lv L, Liu X, Yin C, Yan H, Yuan J. 2016. Effect of Bacillus subtilis CGMCC 1.1086 on the growth performance and intestinal microbiota of broilers. J Appl Microbiol. 120(1):195204. doi:10.1111/jam.12972.

Liu YJ, Liu SJ, Drake HL, Horn MA. 2011. Alphaproteobacteria dominate active 2-methyl-4chlorophenoxyacetic acid herbicide degraders in agricultural soil and drilosphere. Environ Microbiol. 13(4):991-1009. doi:10.1111/j.14622920.2010.02405.x.

Magurran AE. 2004. Measuring biological diversity. Oxford: Blackwell.

Mandl JN, Ahmed R, Barreiro LB, Daszak P, Epstein JH, Virgin HW, Feinberg MB. 2015. Reservoir host immune responses to emerging zoonotic viruses. Cell. 160(1-2):20-35. doi:10.1016/j.cell.2014.12.003.

McDonald D, Price MN, Goodrich J, Nawrocki EP, Desantis TZ, Probst A, Andersen GL, Knight R, Hugenholtz P. 2012. An improved Greengenes taxonomy with explicit ranks for ecological and evolutionary analyses of bacteria and archaea. ISME J 6(3):610 618. doi:10.1038/ismej.2011.139.

Pan D, Yu Z. 2014. Intestinal microbiome of poultry and its interaction with host and diet. Gut Microbes. 5(1):108-119. doi:10.4161/gmic.26945.

Phuong DQ, Dung NT, Jørgensen PH, Handberg KJ, Vinh NT, Christensen JP. 2011. Susceptibility of Muscovy (Cairina Moschata) and mallard ducks (Anas Platyrhynchos) to experimental infections by different genotypes of $\mathrm{H} 5 \mathrm{~N} 1$ avian influenza viruses. Vet Microbiol. 148(2-4):168-174. doi:10.1016/j.vetmic.2010.09.007.

Pielou EC. 1966. The measurement of diversity in different types of biological collections. J Theor Biol. 13:131-144. doi:10.1016/0022-5193(66)90013-0.

Shannon CE, Weaver W. 1949. The Mathematical Theory of Communication. Champaign, IL.

Simpson EH. 1949. Measurement of Diversity. Nature. 163(1):688. doi:10.1038/163688a0.

Singh KM, Shah TM, Reddy B, Deshpande S, Rank DN, Joshi CG. 2014. Taxonomic and gene-centric metagenomics of the fecal microbiome of low and high feed conversion ratio (FCR) broilers. J Appl Genet. 55(1):145-154. doi:10.1007/s13353-013-0179-4.

Susanti R, Fibriana F, Sasi FA. 2018. Genotype of ja- 
vanese backyard waterfowl based on antiviral myxovirus gene. Warasan Songkhla Nakharin. 40(3):498505. doi:10.14456/sjst-psu.2018.74.

Thomas F, Hehemann JH, Rebuffet E, Czjzek M, Michel G. 2011. Environmental and gut Bacteroidetes: The food connection. Front Microbiol. 2(1):1-16. doi:10.3389/fmicb.2011.00093.

Wang W, Cao J, Yang F, Wang X, Zheng S, Sharshov K, Li L. 2016. High-throughput sequencing reveals the core gut microbiome of Bar-headed goose (Anser indicus) in different wintering areas in Tibet. Microbiologyopen. 5(2):287-295. doi:10.1002/mbo3.327.

Yamak US, Sarica M, Boz MA, Ucar A. 2016. The effect of production system (barn and freerange), slaughtering age and gender on carcass traits and meat quality of partridges (Alectoris chukar). Br Poult Sci. 57(2):185-192. doi:10.1080/00071668.2016.1144920.

Yang H, Xiao Y, Gui G, Li J, Wang J, Li D. 2018. Microbial community and short-chain fatty acid profile in gastrointestinal tract of goose. Poult Sci. 97(4):14201428. doi:10.3382/ps/pex438.

Yarza P, Yilmaz P, Pruesse E, Glöckner FO, Ludwig W, Schleifer KH, Whitman WB, Euzéby J, Amann R, Rosselló-Móra R. 2014. Uniting the classification of cultured and uncultured bacteria and archaea using 16S rRNA gene sequences. Nat Rev Microbiol. 12(9):635-645. doi:10.1038/nrmicro3330.

Yeoman CJ, Chia N, Jeraldo P, Sipos M, Goldenfeld ND, White BA. 2012. The microbiome of the chicken gastrointestinal tract. Anim Health Res Rev. 13(1):8999. doi:10.1017/S1466252312000138.

Zhao Y, Li X, Sun S, Chen L, Jin J, Liu S, Song X, Wu C, Lu L. 2019. Protective role of dryland rearing on netting floors against mortality through gut microbiota-associated immune performance in Shaoxing ducks. Poult Sci. 98(10):4530-4538. doi:10.3382/ps/pez268.

Zheng A, Luo J, Meng K, Li J, Bryden WL, Chang W, Zhang S, Wang LX, Liu G, Yao B. 2016. Probiotic (Enterococcus faecium) induced responses of the hepatic proteome improves metabolic efficiency of broiler chickens (Gallus gallus). BMC Genomics. 17(1):1-12. doi:10.1186/s12864-016-2371-5.

Zhou JY, Shen HG, Chen HX, Tong GZ, Liao M, Yang HC, Liu JX. 2006. Characterization of a highly pathogenic H5N1 influenza virus derived from barheaded geese in China. J Gen Virol. 87(7):1823-1833. doi:10.1099/vir.0.81800-0.

Zhu C, Song W, Tao Z, Liu H, Zhang S, Xu W, Li H. 2020. Analysis of microbial diversity and composition in small intestine during different development times in ducks. Poult Sci. 99(2):1096-1106. doi:10.1016/j.psj.2019.12.030. 